\title{
On the Reproducibility of Inversion Recovery Intravoxel Incoherent Motion Imaging in Cerebrovascular Disease
}

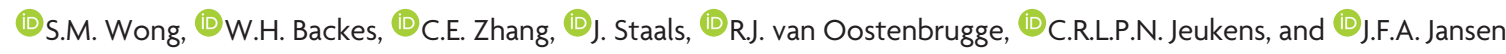

\begin{abstract}
BACKGROUND AND PURPOSE: Intravoxel incoherent motion imaging can measure both microvascular and parenchymal abnormalities simultaneously. The contamination of CSF signal can be suppressed using inversion recovery preparation. The clinical feasibility of inversion recovery-intravoxel incoherent motion imaging was investigated in patients with cerebrovascular disease by studying its reproducibility.
\end{abstract}

MATERIALS AND METHODS: Sixteen patients with cerebrovascular disease (66 \pm 8 years of age) underwent inversion recovery-intravoxel incoherent motion imaging twice. The reproducibility of the perfusion volume fraction and parenchymal diffusivity was calculated with the coefficient of variation, intraclass correlation coefficient, and the repeatability coefficient. ROIs included the normal-appearing white matter, cortex, deep gray matter, white matter hyperintensities, and vascular lesions.

RESULTS: Values for the perfusion volume fraction ranged from 2.42 to $3.97 \times 10^{-2}$ and for parenchymal diffusivity from 7.20 to $9.11 \times 10^{-4}$ $\mathrm{mm}^{2} / \mathrm{s}$, with higher values found in the white matter hyperintensities and vascular lesions. Coefficients of variation were $<3.70 \%$ in normal-appearing tissue and $<9.15 \%$ for lesions. Intraclass correlation coefficients were good to excellent, showing values ranging from 0.82 to 0.99 in all ROIs, except the deep gray matter and cortex, with intraclass correlation coefficients of 0.66 and 0.54 , respectively. The repeatability coefficients ranged from 0.15 to $0.96 \times 10^{-2}$ and 0.10 to $0.37 \times 10^{-4} \mathrm{~mm}^{2} / \mathrm{s}$ for perfusion volume fraction and parenchymal diffusivity, respectively.

CONCLUSIONS: Good reproducibility of inversion recovery-intravoxel incoherent motion imaging was observed with low coefficients of variation and high intraclass correlation coefficients in normal-appearing tissue and lesion areas in cerebrovascular disease. Good reproducibility of inversion recovery-intravoxel incoherent motion imaging in cerebrovascular disease is feasible in monitoring disease progression or treatment responses in the clinic.

ABBREVIATIONS: $C V$ = coefficient of variation; $D=$ parenchymal diffusivity; $f=$ perfusion volume fraction; $I C C=$ intraclass correlation coefficient; $I R=$ inversion recovery; IVIM = intravoxel incoherent motion; NAWM = normal-appearing white matter; $R C=$ repeatability coefficient

$\mathbf{P}$ erfusion imaging using an exogenous contrast agent is commonly used in the clinic in patients with cerebrovascular diseases. ${ }^{1,2}$ This technique is sensitive to the microcirculatory con-

Received June 4, 2017; accepted after revision October 3.

From the Departments of Radiology and Nuclear Medicine (S.M.W., W.H.B., C.R.L.P.N.J., J.F.A.J.), Neurology (C.E.Z., J.S., R.J.v.O.), School for Mental Health and Neuroscience (S.M.W., W.H.B., C.E.Z., R.J.v.O., J.F.A.J.), and Cardiovascular Research Institute Maastricht (C.E.Z., J.S., R.J.v.O.), Maastricht University Medical Centre, Maastricht, the Netherlands.

This project was funded by the Netherlands Organization for Scientific Research, (grant: 017.009.048), Hersenstichting (grant: 2013(1)-195), and Stichting de Weijerhorst Foundation.

Please address correspondence to J.F.A. Jansen, PhD, Department of Radiology and Nuclear Medicine, Maastricht University Medical Centre, PO Box 5800, 6202 AZ, Maastricht, the Netherlands; e-mail: jacobus.jansen@mumc.nl; @jfajansenphd

- Indicates open access to non-subscribers at www.ajnr.org

http://dx.doi.org/10.3174/ajnr.A5474 dition and provides insight into the cerebral hemodynamics in, for example, patients with ischemic stroke. Because this technique is contraindicated in patients with impaired renal function, there is interest in techniques that avoid contrast agents. For example, by labeling endogenous blood as a tracer, arterial spin-labeling has been used to measure perfusion. However, this technique is associated with low signal-to-noise ratios and is dependent on the arterial transit time. Changes in flow conditions due to disease (eg, stroke) can complicate selecting the correct arterial transit time. ${ }^{3}$ Another technique without the use of a contrast agent that has received attention is intravoxel incoherent motion (IVIM) imaging. IVIM is a DWI technique that measures the diffusion properties of water molecules in static tissue and in blood flowing into the capillary networks. Therefore, it can measure perfusion and diffusion properties in the brain simultaneously. ${ }^{4}$ The resulting perfusion volume fraction $(f)$ and parenchymal diffusivity $(D)$ 
provide information on the microvasculature and parenchymal microstructure, respectively, and have shown promising results in assisting in the assessment of stroke. ${ }^{5,6}$ Moreover, with this technique, the affected microvasculature and parenchyma in patients with cerebral small-vessel disease have been demonstrated previously. ${ }^{7}$

Unfortunately, the IVIM signal and the resulting measures can be affected by partial volume effects of CSF. The contamination of CSF has been previously demonstrated to be relevant for DWI, and an improved accuracy of diffusion measures was shown when suppressing the CSF signal. ${ }^{8,9}$ Recently, this suppression has been applied to IVIM. ${ }^{10}$ This so-called inversion recovery-based IVIM technique (ie, IR-IVIM) can be particularly helpful for patients with cerebrovascular disease because these patients often have brain atrophy, ${ }^{11}$ which can increase the CSF contamination.

The reproducibility of IR-IVIM has not yet been fully studied. Several groups have investigated the reproducibility of conventional IVIM and have shown good results (eg, low coefficients of variation $[\mathrm{CVs}]$ ) for both the perfusion volume fraction and parenchymal diffusivity. ${ }^{12-15}$ Thus far, only 1 reproducibility study has been performed on IR-IVIM. This study examined only $f$ and healthy participants. ${ }^{10}$

We aimed to assess the clinical feasibility of IR-IVIM using the reproducibility of the perfusion volume fraction and parenchymal diffusivity.

\section{MATERIALS AND METHODS Study Population}

For this study, we enrolled patients with varying manifestations of cerebrovascular disease $(n=17): 1)$ cerebral small vessel disease $(n=11), 2)$ cortical stroke $(n=5)$, and 3$)$ intracerebral hemorrhage $(n=1)$. Patients were recruited in either the transient ischemic attack outpatient service or the stroke unit of the department of neurology of the hospital. A more detailed description of the study population has been published previously. ${ }^{16}$

All patients were scanned twice on separate days. The patient with intracerebral hemorrhage did not complete both sessions and was excluded from analysis. This exclusion resulted in 16 patients ( 14 men and 2 women; mean age, $66 \pm 8$ years; $1.9 \pm 2.2$ mean days between the 2 sessions) suitable for image analysis.

\section{Standard Protocol Approvals, Registrations, and Patient Consents}

The medical ethics committee of our institution approved this study. Written informed consent was obtained from patients before participation.

\section{Imaging Protocol}

Participants underwent brain imaging on an MR imaging system (3T, Achieva TX; Philips Healthcare, Best, the Netherlands) using a 32-element head coil suitable for parallel imaging. For IVIM imaging, a Stejskal-Tanner DWI single-shot spin-echo EPI pulse sequence was used (TR/TE, 6800/84 ms; FOV, $221 \times 269 \times 139$ $\mathrm{mm}^{3} ; 2.4-\mathrm{mm}$ cubic voxel size). To suppress the CSF signal, an inversion pulse (TI, $2230 \mathrm{~ms}$ ) was given before the DWI sequence. ${ }^{10}$ The total scan time of IVIM imaging using the inversion pulse was 14 minutes. Fifteen DWIs were acquired in 3 orthogo- nal directions using multiple diffusion-sensitive b-values $(0,5,7$, $10,15,20,30,40,50,60,100,200,400,700$, and $\left.1000 \mathrm{~s} / \mathrm{mm}^{2}\right)$. IVIM images were aligned with the corpus callosum (ie, the line connecting the center of the genu and splenium of the corpus callosum). The number of signal averages for the highest $2 \mathrm{~b}$-values was 2 and 3 , instead of 1 , respectively, to increase the signalto-noise ratio at high b-values.

For anatomic reference, a T1-weighted sequence (TR/TI/TE, 8.3/800/3.8 ms; FOV, $256 \times 256 \times 160 \mathrm{~mm}^{3}$; cubic voxel, 1.0 $\mathrm{mm}^{3}$ ) and T2-weighted FLAIR sequence (TR/TI/TE, 4800/1650/ $299 \mathrm{~ms}$; FOV, $250 \times 256 \times 180 \mathrm{~mm}^{3}$; cubic voxel, $1.0 \mathrm{~mm}^{3}$ ) were performed.

\section{Image Analysis}

Brain Segmentation. T1-weighted images were segmented into white matter, cortex, and deep gray matter using FreeSurfer software (http://surfer.nmr.mgh.harvard.edu) ${ }^{17}$ and FSL (Version 5.0; http://www.fmrib.ox.ac.uk/fsl). ${ }^{18}$ Subsequently, the normalappearing white matter (NAWM) was separated from the white matter hyperintensities on FLAIR using a semiautomated segmentation algorithm ${ }^{19}$ followed by visual checks. Visible vascular lesions were identified $(n=4)$ by a neuroradiologist ( $>20$ years of experience). Because a range of cerebrovascular diseases was included (ie, cerebral small-vessel disease and cortical infarction), we explored various ROIs: NAWM, cortex, deep gray matter, white matter hyperintensities, and vascular lesions (Fig 1A,-B). For patients $(n=5)$ with an acute infarct, only the contralateral hemisphere was considered for analysis.

IVIM Analysis. IVIM images were corrected for distortions and head displacements by registering to a corresponding T1weighted image, using ExploreDTI Version 4.8.3 (http:// exploredti.com/). ${ }^{20}$ Subsequently, trace images were obtained by calculating the geometric mean of the DWIs measured in 3 orthogonal directions. Henceforth, spatial smoothing was performed on the trace images with a 3-mm (full width at half maximum) Gaussian kernel. The signal-to-noise ratio $^{21}$ of cerebral tissue of the trace images was 60; and of the images obtained in 1 direction, it was 45 with a b-value of $1000 \mathrm{~s} / \mathrm{mm}^{2}$, which was higher than the recommended value (ie, 30) for reliable IVIM estimation. ${ }^{22}$

Model Fitting. We used a 2-compartment model representing a vascular and nonvascular component, which also accounts for CSF contamination and differences in the relaxation time of blood and tissue. ${ }^{7}$ The IVIM signal was fitted biexponentially using a 2-step approach, which has been described previously ${ }^{7,23,24}$ : 1) The parenchymal diffusivity was obtained by fitting a monoexponential decay to the signal attenuation curve, obtained with b-values of $>200 \mathrm{~s} / \mathrm{mm}^{2}$; and 2) a biexponential decay was fitted to the signal attenuation curve, using all b-values and a fixed $D$, to obtain the perfusion volume fraction. This fitting was performed in a voxelwise manner; and for each ROI, the average IVIM measure was calculated. This calculation was performed with in-house software in Matlab R2012a (MathWorks, Natick, Massachusetts).

AJNR Am J Neuroradiol 39:226-31 Feb 2018 www.ajnr.org 

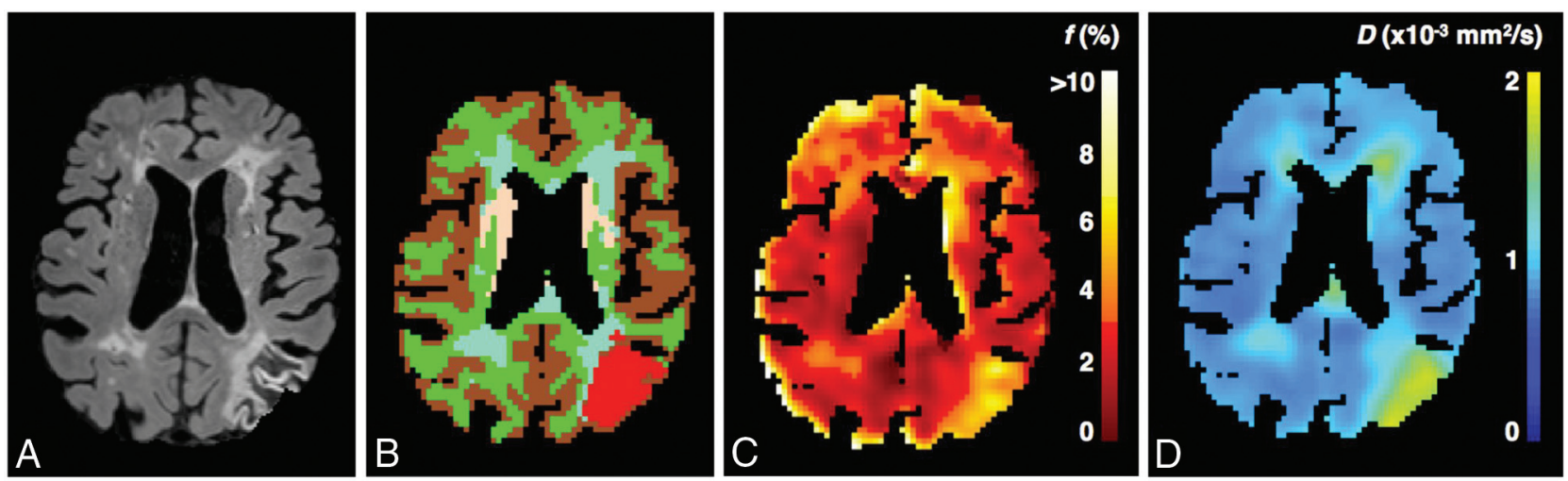

FIG 1. A, FLAIR image of a patient with a cortical infarct. Cerebral tissue was segmented (B) into normal-appearing white matter (green), cortex (brown), deep gray matter (pink), white matter hyperintensities (light blue), and vascular lesion (red). $C$ and $D$, The calculated perfusion volume fraction map and parenchymal diffusivity map are shown, respectively.

\section{Reproducibility Assessment and Statistical Analysis}

All IVIM measures obtained with the trace images were calculated for 2 sessions and compared between sessions to evaluate the reproducibility using the coefficient of variation $(\mathrm{CV})$, the repeatability coefficient (RC), and the intraclass correlation coefficient (ICC). ${ }^{25}$ The CV expresses the relative variation within the subject from 1 session to the second session, whereas the ICC provides information on the effect of this variation with respect to the biologic variation of the study population. Furthermore, the RC indicates the minimal difference needed between the 2 measurements obtained from the same method to be considered an actual change rather than a test-retest error. ${ }^{26}$

$\mathrm{CV}$ was calculated to express the relative within-subject variation and is defined as

$$
\mathrm{CV}=\frac{\sigma_{\mathrm{ws}}}{\mu_{\mathrm{ws}}} \times 100 \%
$$

where $\sigma_{\mathrm{ws}}$ is the population SD during 2 sessions within the same subject and $\mu_{\mathrm{ws}}$ the mean value during 2 sessions within the same subject.

The repeatability coefficient was calculated as

$$
\mathrm{RC}=1.96 \times \sqrt{2} \times \sigma_{\mathrm{ws}} .
$$

Both the CV and RC were calculated per participant and averaged over all subjects to represent the overall CV and RC. Low CV and RCs represented good test-retest reproducibility.

Third, the intraclass correlation coefficient was used, defined as

$$
\mathrm{ICC}=\frac{\sigma_{\mathrm{bs}}{ }^{2}}{\sigma_{\mathrm{bs}}{ }^{2}+\sigma_{\mathrm{ws}}{ }^{2}},
$$

where $\sigma_{\mathrm{bs}}{ }^{2}$ denotes the biologic variance, which was obtained by calculating the variance over the average values of both sessions in all patients. The ICC expresses the effect of the biologic variance on the total variance rather than the test-retest error $\left(\sigma_{\mathrm{ws}}{ }^{2}\right)$. ICC values toward 1 represent good reproducibility. The ICC was categorized as follows: poor, ICC $<0.40$; moderate, $0.40 \leq$ ICC $<$ 0.60 ; good, $0.60 \leq$ ICC $<0.75$; and excellent, $0.75 \leq$ ICC $\leq$ $1.00 .^{27}$
Reproducibility values of the perfusion fraction and parenchymal diffusivity

\begin{tabular}{lccccc}
\hline Region & $\begin{array}{c}\text { Session 1 } \\
\text { (mean) } \\
\text { (SD) }\end{array}$ & $\begin{array}{c}\text { Session 2 } \\
\text { (mean) } \\
\text { (SD) }\end{array}$ & $\begin{array}{c}\text { CV } \\
\text { (\%) }\end{array}$ & RC $^{\mathbf{b}}$ & ICC \\
\hline$f \quad$ & & & & & \\
NAWM & $2.42(0.23)$ & $2.42(0.22)$ & 2.27 & 0.15 & 0.86 \\
Cortex & $2.79(0.37)$ & $2.76(0.38)$ & 2.89 & 0.21 & 0.89 \\
DGM & $2.90(0.27)$ & $2.81(0.31)$ & 3.70 & 0.29 & 0.66 \\
WMH & $3.22(0.33)$ & $3.23(0.31)$ & 2.47 & 0.22 & 0.84 \\
VL & $3.46(0.79)$ & $3.97(1.33)$ & 9.15 & 0.96 & 0.82 \\
DL & & & & & \\
NAWM & $7.20(0.19)$ & $7.27(0.22)$ & 0.49 & 0.10 & 0.88 \\
Cortex & $7.35(0.12)$ & $7.44(0.17)$ & 0.68 & 0.14 & 0.54 \\
DGM & $7.36(0.28)$ & $7.42(0.27)$ & 0.46 & 0.09 & 0.95 \\
WMH & $9.02(0.64)$ & $9.11(0.69)$ & 0.70 & 0.18 & 0.97 \\
VL & $8.83(1.88)$ & $9.09(2.10)$ & 1.30 & 0.37 & 0.99 \\
\hline
\end{tabular}

Note:-DGM indicates deep gray matter; WMH, white matter hyperintensities; $\mathrm{VL}$, vascular lesion.

${ }^{a}$ Mean and SD were calculated for all participants.

${ }^{b}$ Units: $\times 10^{-2}(f) ; \times 10^{-4} \mathrm{~mm}^{2} / \mathrm{s}(D)$.

\section{Effect of Smoothing}

To analyze the effect of smoothing on the reproducibility, we smoothed DWIs with a Gaussian kernel with increasing full width at half maximum ranging from 0 to $6 \mathrm{~mm}$. Subsequently, $\mathrm{CV}$ values were calculated and used for comparing the reproducibility for increased smoothing. This calculation was performed for both $f$ and $D$ and for the ROIs in the white and gray matter.

\section{RESULTS}

The Table shows the results of the reproducibility of the IVIM parameter perfusion volume fraction and parenchymal diffusivity. Figure $1 C,-D$ shows examples of $f$ and $D$ maps. The measured $f\left(2.42-2.90 \times 10^{-2}\right)$ and $D\left(7.20-7.44 \times 10^{-4} \mathrm{~mm}^{2} / \mathrm{s}\right)$ seen in patients with cerebrovascular disease are in the range of expected values in NAWM and gray matter. White matter hyperintensities and vascular lesions had values for $f$ and $D$ ranging from 3.22$3.97 \times 10^{-2}$ and $8.83-9.11 \times 10^{-4} \mathrm{~mm}^{2} / \mathrm{s}$, respectively. CVs of $\leq 3.7 \%$ for $f$ and $D$ were observed for the white and gray matter. Vascular lesions showed relatively higher CVs for $f$ and $D$ of 9.15 and $1.30 \%$, respectively. Excellent ICCs $(0.82-0.97)$ were observed for both IVIM parameters, except for $f$ in the deep gray matter and $D$ in the cortex showing moderate-to-good ICCs. Fur- 


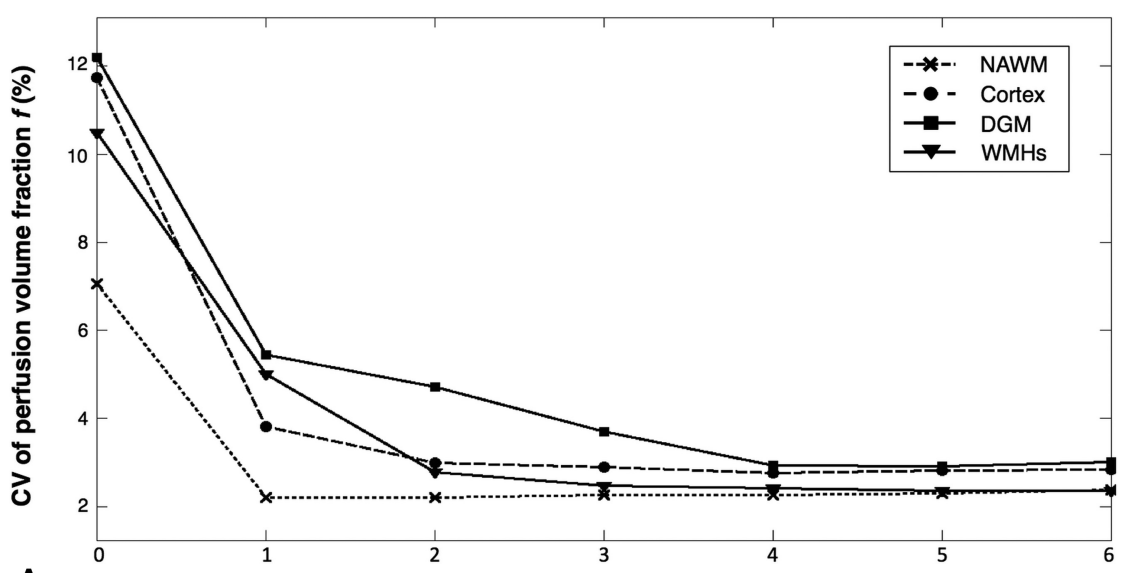

A

Full width half maximum of smoothing kernel $(\mathrm{mm})$

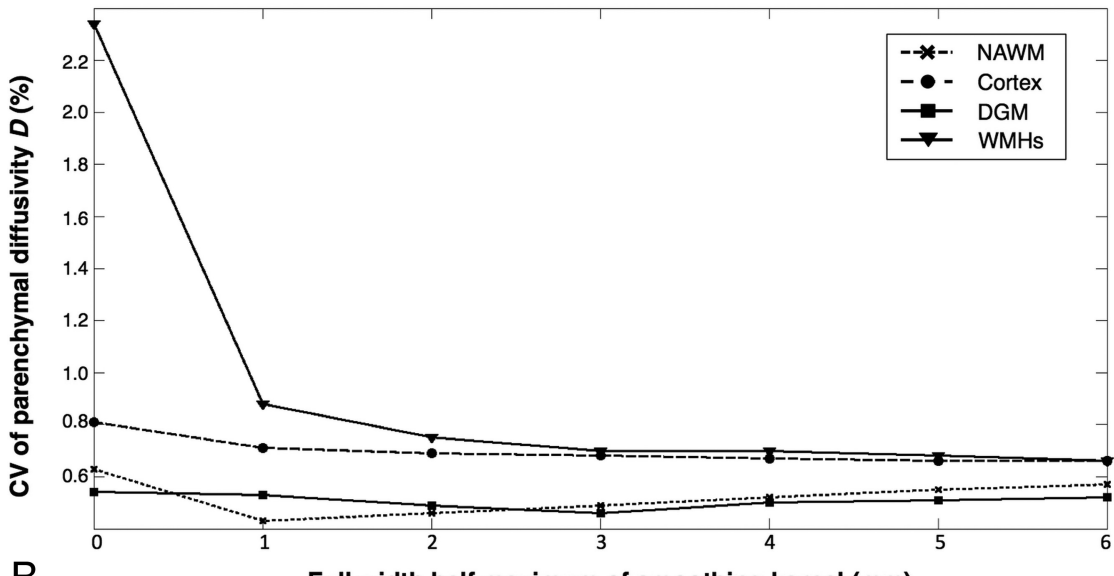

B

Full width half maximum of smoothing kernel $(\mathrm{mm})$

FIG 2. Coefficients of variation as a function of the full width at half maximum of the smoothing kernels ranging from 0 to $6 \mathrm{~mm}$ for the perfusion volume fraction $(A)$ and parenchymal diffusivity (B). Higher CVs are present when no smoothing was performed. CVs decreased up to 3 times when smoothing was applied.

thermore, RCs ranged from $0.15-0.96 \times 10^{-2}$ for $f$ and from $0.09-0.37 \times 10^{-4} \mathrm{~mm}^{2} / \mathrm{s}$ for $D$.

When comparing our CVs obtained with smoothing (ie, full width at half maximum $=3 \mathrm{~mm}$ ) with CVs without smoothing (ie, full width at half maximum $=0 \mathrm{~mm}$ ), we observed lower CVs for $f(\mathrm{CV}, 2.27 \%-3.70 \%)$ when smoothing was applied than when no smoothing was applied (CV, 7.05\%-12.20\%) in the white and gray matter. For $D$, lower CVs were also found when smoothing was applied $(\mathrm{CV}, 0.49 \%-0.70 \%)$ than without smoothing $(\mathrm{CV}$, $0.54 \%-2.34 \%)$ in the white and gray matter. In Fig 2, the CVs for an increasing size of the smoothing kernel are shown. For $f$, CVs without smoothing ranged from 7.05\%-12.20\%, and with smoothing, from $2.20 \%-5.45 \%$ in the white and gray matter. For $D$, CVs without smoothing ranged from $0.54 \%-2.34 \%$, and with smoothing, from $0.43 \%-0.88 \%$ in the white and gray matter.

\section{DISCUSSION}

In this study, we assessed the reproducibility of IR-IVIM. Low CVs and moderate-to-excellent ICCs were observed for both normal-appearing tissue and lesion areas. Furthermore, low values for RCs were observed for all regions.

Several groups have evaluated the reproducibility of conventional IVIM in the brain. ${ }^{12-15}$ In patients with gliomas, CVs of
$6.5 \%$ and $0.6 \%$ in the NAWM outside the tumor mass for $f$ and $D$ were reported, respectively. ${ }^{12}$ Furthermore, Peterson et $\mathrm{al}^{15}$ investigated healthy participants and reported moderate-to-excellent ICCs for $f$ in the deep gray matter. In a multicenter study ${ }^{14}$ also in healthy participants, CVs of $f$ were $5.8 \%$ and $11.1 \%$, and CVs of $D$ were $1.7 \%$ and $2.1 \%$ in the white matter and gray matter, respectively. In comparison with these results, we reported somewhat lower CVs of $f$ and $D$ for the white and gray matter, not exceeding $3.70 \%$ and $0.70 \%$, respectively, and comparable ICCs. An explanation for the lower CV values might be the effect of smoothing. We have shown that our CVs with smoothing (ie, full width at half maximum $=3 \mathrm{~mm}$ ) were generally 3 times lower than when no smoothing was applied. When one assesses CVs with no smoothing $(f, \mathrm{CV} \leq 12.20 \%$; and $D$, $\mathrm{CV} \leq 2.34 \%$ ), it can be observed that CVs are in the same range as in the other studies (ie, $f, 5.8 \%-11.1 \%$; and $D, 0.6 \%-$ $\left.2.1 \%^{12,15,14}\right)$. This finding indicates that the reproducibility of IR-IVIM is like that of conventional IVIM. Nevertheless, differences exist between our study and other studies that can influence the accurate comparison between IR-IVIM and IVIM, including the study population (ie, healthy controls ${ }^{14,15}$ versus patients with disease and cerebrovascular disease versus brain glio$\operatorname{mas}^{12}$ ); the exact method used (eg, IVIM versus IR-IVIM and selection of ROIs to analyze the reproducibility); and image-acquisition settings, which can contribute to a better signal-to-noise ratio (eg, the number of averages and the number of diffusionsensitizing directions).

A study that used a similar IR-IVIM technique also explored the reproducibility of $f$ in 5 healthy adults at $1.5 \mathrm{~T}$ in the gray matter. ${ }^{10}$ They reported high-repeatability indices (dissimilar to the repeatability coefficient but comparable with $\mathrm{CV}$ ) ranging from $27.3 \%$ to $65.2 \%$, with a smoothing window full width at half maximum of $0-6 \mathrm{~mm}$. The seemingly higher reproducibility we show from our CVs (ie, full width at half maximum, 0-6 mm: CVs, $2.77 \%-12.20 \%$ ) may be explained by several factors: First, our study was performed at a higher field strength (ie, $3 \mathrm{~T}$ versus 1.5T). At a higher field strength, a better signal-to-noise ratio and/or a higher spatial resolution can be acquired, which may contribute to better reproducibility. Second, the methods used to calculate the reproducibility values were different. Whereas we have calculated reproducibility values from the mean IVIM measure taken over an ROI (ie, "mean method"), they calculated the reproducibility values in a voxelwise manner. An earlier study has shown that CVs calculated in a voxelwise manner are approxi- 
mately twice as high as CVs obtained with the mean method. ${ }^{25}$ On the basis of this observation, our CV values of $f$ for the voxelwise method can be roughly estimated to be approximately $\leq 7.40 \%$ for the white and gray matter, which is lower than that reported by Hales and Clark $(27.3 \%-65.2 \%)^{10}$ and is indicative of good reproducibility.

In our study, we applied spatial smoothing, which is beneficial because it improves the signal-to-noise ratio and corrects for small movement or spatial registration errors. We observed that smoothing might lead to improved CVs ( $f, 2.20 \%-5.45 \% ; D$, $0.43 \%-0.88 \%$ ), which were generally up to 3 times lower compared with CVs without smoothing $(f, 7.05 \%-12.20 \%$; $D, 0.54 \%-$ $2.34 \%)$. This finding is in accordance with a previous study that reported better reproducibility with an increasing full width at half maximum of the smoothing kernel. ${ }^{10}$

Lower ICCs in the cortex and DGM can be observed, which indicate lower reproducibility in those areas. We argue that partial volume effects in general can still contribute to the lower reproducibility values. Even though CSF is suppressed and contamination of CSF is reduced, other tissue (eg, white matter) can still contribute to partial volume effects leading to lower reproducibility values.

The clinical contribution of IVIM in neuroimaging is an active research topic. IR-IVIM shows good reproducibility in both normal-appearing tissue and lesion area, which is required when monitoring disease progression or treatment responses. The clinical applicability of our reproducibility results can be demonstrated by the following: A difference in $f$ and $D$ in the stroke region $\left(f, 2.6 \% \pm 1.9 \% ; D, 3.9 \pm 0.79 \times 10^{-4} \mathrm{~mm}^{2} / \mathrm{s}\right)$ compared with the contralateral side $(f, 5.6 \% \pm 2.5 \% ; D$, versus $7.5 \% \pm$ $0.86 \times 10^{-4} \mathrm{~mm}^{2} / \mathrm{s}$ ) has been reported. ${ }^{6}$ Our RC values in the vascular lesions of 1.0 and 0.4 for $f$ and $D$, respectively, show that this difference can be easily detected with IR-IVIM. Furthermore, another benefit of IR-IVIM, as opposed to contrast-enhanced techniques, is that it can be performed multiple times to assess both diffusion and perfusion properties without the concern of contrast deposition in the brain. In addition, the advantage of DWI in ischemic stroke has already been widely reported, ${ }^{2}$ and the IR-IVIM-derived $D$ was corrected for microvascular contributions; this process resulted in a more accurate $D$ (ie, less biased).

The strength of this study is that it was performed in a patient group in which both normal-appearing tissue and lesion areas (ie, white matter hyperintensities and vascular lesions) were investigated. On the contrary, in this study, only 2 sessions were performed, whereas multiple scan sessions could have improved the reproducibility evaluation. Furthermore, IVIM imaging without suppression of the CSF would have provided a more accurate comparison of reproducibility between IVIM with and without CSF suppression. However, IVIM without CSF suppression was not acquired because there was limited scan time. In addition, only a few patients $(n=4)$ had an acute infarct that we could use to investigate the vascular lesion area. However, we were able to provide some preliminary results. Last, in this study, we assumed a random microvascular network and hence an isotropic $f$. However, in a recent study, $f$ was shown to be anisotropic in the gray matter. ${ }^{28}$ Further investigation is needed to study the effect on the reproducibility of fitting the anisotropic $f$.

\section{CONCLUSIONS}

IR-IVIM shows good reproducibility in its clinical feasibility in patients with cerebrovascular disease. Good reproducibility is a primary requirement in monitoring disease progression or treatment responses in the clinical setting.

\section{ACKNOWLEDGMENTS}

The authors greatly appreciate the effort and help of Professor P. Hofman in evaluating MR images on vascular lesions.

Disclosures: Sau May Wong_RELATED: Other: Stichting de Weijerhorst, Comments: Salary. Walter H. Backes—RELATED: Grant: Stichting de Weijerhorst*. *Money paid to the institution.

\section{REFERENCES}

1. Wintermark M, Sesay M, Barbier E, et al. Comparative overview of brain perfusion imaging techniques. J Neuroradiol 2005;32:294-314 CrossRef Medline

2. Latchaw RE, Alberts MJ, Lev MH, et al; American Heart Association Council on Cardiovascular Radiology and Intervention, Stroke Council, and the Interdisciplinary Council on Peripheral Vascular Disease. Recommendations for imaging of acute ischemic stroke: a scientific statement from the American Heart Association. Stroke 2009;40:3646-78 CrossRef Medline

3. Alsop DC, Detre JA, Golay X, et al. Recommended implementation of arterial spin-labeled perfusion MRI for clinical applications: a consensus of the ISMRM perfusion study group and the European consortium for ASL in dementia. Magn Reson Med 2015;73:102-16 CrossRef Medline

4. Le Bihan D, Breton E, Lallemand D, et al. Separation of diffusion and perfusion in intravoxel incoherent motion MR imaging. Radiology 1988;168:497-505 Medline

5. Yao Y, Zhang S, Tang X, et al. Intravoxel incoherent motion diffusion-weighted imaging in stroke patients: initial clinical experience. Clin Radiol 2016;71:938.e11-16 CrossRef

6. Federau C, Sumer S, Becce F, et al. Intravoxel incoherent motion perfusion imaging in acute stroke: initial clinical experience. $\mathrm{Neu}$ roradiology 2014;56:629-35 CrossRef Medline

7. Wong SM, Eleana Zhang C, van Bussel FC, et al. Simultaneous investigation of microvasculature and parenchyma in cerebral small vessel disease using intravoxel incoherent motion imaging. Neuroimage Clin 2017;14:216-21 CrossRef Medline

8. Latour LL, Warach S. Cerebral spinal fluid contamination of the measurement of the apparent diffusion coefficient of water in acute stroke. Magn Reson Med 2002;48:478-86 CrossRef Medline

9. Bhagat YA, Beaulieu C. Diffusion anisotropy in subcortical white matter and cortical gray matter: changes with aging and the role of CSF-suppression. J Magn Reson Imaging 2004;20:216-27 CrossRef Medline

10. Hales PW, Clark C. Combined arterial spin labeling and diffusionweighted imaging for noninvasive estimation of capillary volume fraction and permeability-surface product in the human brain. J Cereb Blood Flow Metab 2013;33:67-75. CrossRef Medline

11. Nitkunan A, Lanfranconi S, Charlton RA, et al. Brain atrophy and cerebral small vessel disease a prospective follow-up study. Stroke 2011;42:133-38 CrossRef Medline

12. Bisdas S, Koh TS, Roder C, et al. Intravoxel incoherent motion diffusion-weighted MR imaging of gliomas: feasibility of the method and initial results. Neuroradiology 2013;55:1189-96 CrossRef Medline

13. Rydhög A, van Osch MJP, Lindgren E, et al. Intravoxel incoherent motion (IVIM) imaging at different magnetic field strengths: what is feasible? Magn Reson Imaging 2014;32:1247-58 CrossRef Medline 
14. Grech-Sollars M, Hales PW, Miyazaki K, et al. Multi-centre reproducibility of diffusion MRI parameters for clinical sequences in the brain. NMR Biomed 2015;28:468-85 CrossRef Medline

15. Peterson ET, Zahr NM, Kwon D, et al. Intra voxel incoherent motion (IVIM) in brain regions: a repeatability and aging study. In: Proceedings of the International Society for Magnetic Resonance in Medicine 24th Scientific Meeting \& Exhibition, Singapore. May 7-13, 2016: 3410

16. Wong SM, Jansen JFA, Zhang CE, et al. Measuring subtle leakage of the blood-brain barrier in cerebrovascular disease with DCE-MRI: test-retest reproducibility and its influencing factors. J Magn Reson Imaging 2017;46:159-66 CrossRef Medline

17. Fischl B, Salat DH, Busa E, et al. Whole brain segmentation: automated labeling of neuroanatomical structures in the human brain. Neuron 2002;33:341-55 CrossRef Medline

18. Jenkinson M, Bannister P, Brady M, et al. Improved optimization for the robust and accurate linear registration and motion correction of brain images. Neuroimage 2002;17:825-41 CrossRef Medline

19. de Boer R, Vrooman H, van der Lijn F, et al. White matter lesion extension to automatic brain tissue segmentation on MRI. Neuroimage 2009;45:1151-61 CrossRef Medline

20. Leemans A, Jeurissen B, Sijbers J, et al. ExploreDTI: a graphical toolbox for processing, analyzing, and visualizing diffusion MR data. In: Proceedings of the International Society for Magnetic Resonance in Medicine 17th Scientific Meeting \& Exhibition, Honolulu, Hawaii. April 18-24, 2009: 3537

21. The Association of Electrical Equipment and Medical Imaging Manufacturers. Determination of signal-to-noise ratio (SNR) in diagnos- tic magnetic resonance imaging. EMA MS 1-2008 (R2014.) April, 30, 2015. https://www.nema.org/Standards/Pages/Determination-ofSignal-to-Noise-Ratio-in-Diagnostic-Magnetic-Resonance-Imaging. aspx. Accessed September 1, 2017

22. Wu WC, Chen YF, Tseng HM, et al. Caveat of measuring perfusion indexes using intravoxel incoherent motion magnetic resonance imaging in the human brain. Eur Radiol 2015;25:2485-92 CrossRef Medline

23. van Bussel FC, Backes WH, Hofman PA, et al. On the interplay of microvasculature, parenchyma, and memory in type 2 diabetes. $\mathrm{Di}$ abetes Care 2015 38:876-82 CrossRef Medline

24. Federau C, O'Brien K, Meuli R, et al. Measuring brain perfusion with intravoxel incoherent motion (IVIM): initial clinical experience. $J$ Magn Reson Imaging 2014;39:624-32 CrossRef Medline

25. Jansen JFA, Kooi ME, Kessel AG, et al. Reproducibility of quantitative cerebral T2 relaxometry, diffusion tensor imaging, and $1 \mathrm{H}$ magnetic resonance spectroscopy at 3.0 Tesla. Invest Radiol 2007;42: 327-37 CrossRef Medline

26. Bland JM, Altman DG. Measurement error. BMJ 1996;312:1654 CrossRef Medline

27. Cicchetti DV. Guidelines, criteria, and rules of thumb for evaluating normed and standardized assessment instruments in psychology. Psychol Assess 1994;6:284-90 CrossRef

28. Finkenstaedt T, Klarhoefer M, Eberhardt C, et al. The IVIM signal in the healthy cerebral gray matter: a play of spherical and non-spherical components. Neuroimage 2017;152:340-47 CrossRef Medline 